\title{
The Research on Computer Teaching Strategies Based on Network Teaching Mode WebQuest
}

\author{
Lifang Lu ${ }^{1, a}$ \\ ${ }^{1}$ Yunnan Physical Education College of Qujing Normal University, china \\ a molifangfei@126.com
}

Keywords: Computer Teaching Strategies; Network Teaching Mode ; WebQuest

\begin{abstract}
Inquiry learning is a learning that curriculum reform advocates in our country. Network openness, diversity and efficiency provide an unprecedented favorable conditions for network-based inquiry learning. The way to learning and teaching of information technology is undergoing historic change. By constructing "dominant - subject" Teaching mode, while focuses on teachers teaching philosophy, on the other hand fully exploits network resources, and increases the interaction between teachers and students in the teaching process, thus enabling student autonomy and collaboration to get better playing and continuing to study other courses in a good foundation. Based on this, a computer teaching strategies based on network teaching mode WebQuest is studied. This article firstly introduces WebQuest related overview and then bases on WebQuest teaching mode to explore, and by teaching cases related analysis, studies computer teaching strategies based on network teaching mode WebQuest.
\end{abstract}

\section{Introduction}

Since the 1980s, reconstruction of the basic education curriculum based on inquiry learning has gradually become the focus of the world curriculum reform. Inquiry learning needs intensive rich educational resources, needs to fully conversational learning situations, needs to be recorded traces of information tools and convenient place to learn the results show, and the network just to meet these requirements. Therefore, studying inquiry learning based on network environment has become an important issue of people concern. WebQuest mode can be encouraged to enroll through the Internet, direct access to the expert, search the database for the latest reports. And based on analysis, synthesis and evaluation, propose method to solve problems, maximize students' creativity, innovation and capacity for self-learning. Therefore, its use becomes more and more widely. Based on this, a computer teaching strategies based on network teaching mode WebQuest is studied. I hope from this paper's research, can provide a reference for the related people.

\section{WebQuest related overview}

There are two levels of levels WebQuest: short-cycle WebQuest and long-cycle WebQuest. Short-cycle WebQuest is designed for one to three hours of course unit.Its objective is to obtain and integrate the teaching of knowledge. At the end of each short-cycle WebQuest, learners need to handle large amounts of new information and ultimately the formation of knowledge of this information. Long-cycle WebQuest typically lasts from 1 week to a month, in 3 hours or more. Its teaching objectives are to expand and refine knowledge. After completing a longer-cycle WebQuest later, learners knowledge system for in-depth analysis, the ability to convert knowledge and migration in some way. Learners make some available for others to use online or offline interactive multimedia work to prove their understanding of this knowledge.

In order to enable learners to clear learning objectives, avoid any surfing aimlessly, every WebQuest have been carefully designed. It gives learners a clear direction, gives learner an interesting and feasible task, and provide the necessary, able to guide them through the task of resources, but also tell their future evaluation methods, as well as general and ways to further expand the curriculum. A WebQuest must include introduction, task, process, resources, evaluation, conclusion,which is shown as Fig.1: 


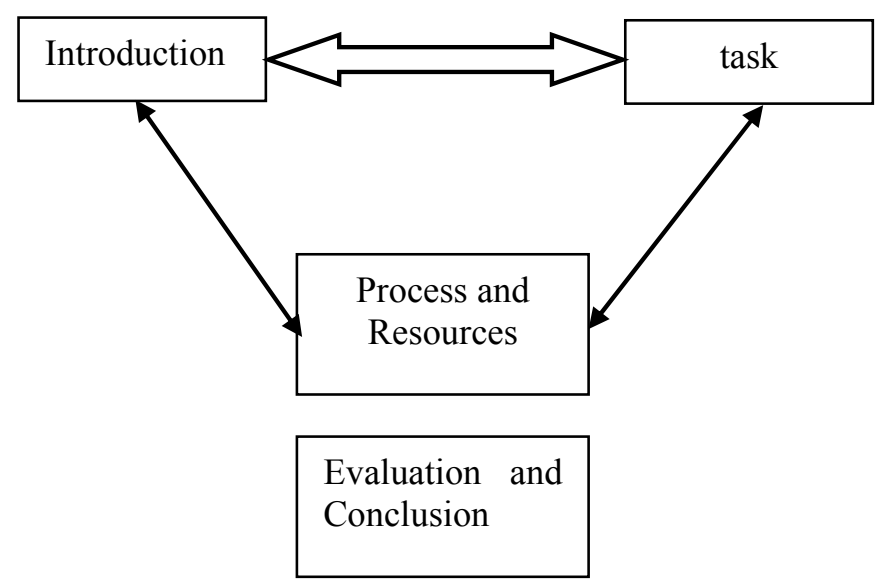

Fig.1 WebQuest structure and composition

In the Fig.1, we can know that introduction need to achieve two purposes: First, give students a clear what they will learn; second, through a variety of ways to enhance students' interest in learning. To this end, the selected topic should be related to the students 'past experience, related to the students' future goals. The creation of introduction must be attractive and interesting.

Tasks section must clarify what kind of results you want to achieve when students complete WebQuest. WebQuest design task for students should have the authenticity, integrity, openness. Process section describes the steps required for students to complete the task. In the WebQuest process module, teachers give advice to learn. Resources section is selected by teachers, will help students complete a list of page tasks, most of the resources as a hyperlink to the information on the World Wide Web.

Such a high level forms of learning based on WebQuest, is a high level in Broome's Taxonomy. From the purpose of the evaluation is to promote student inquiry levels by evaluating the continuous development and improvement. From the evaluation of the focus point of view, should be concerned about students to explore the process of learning performance, whether the inquiry can not be concluded or whether the correct conclusion as the only or the main index. In the concluding part of the whole activity of teachers to lessons learned, to encourage students to reflect on the process, expand and summarize what they have learned, and to encourage students to expand their experience gained in other areas.

WebQuest Mode Figure is shown as Fig.2.

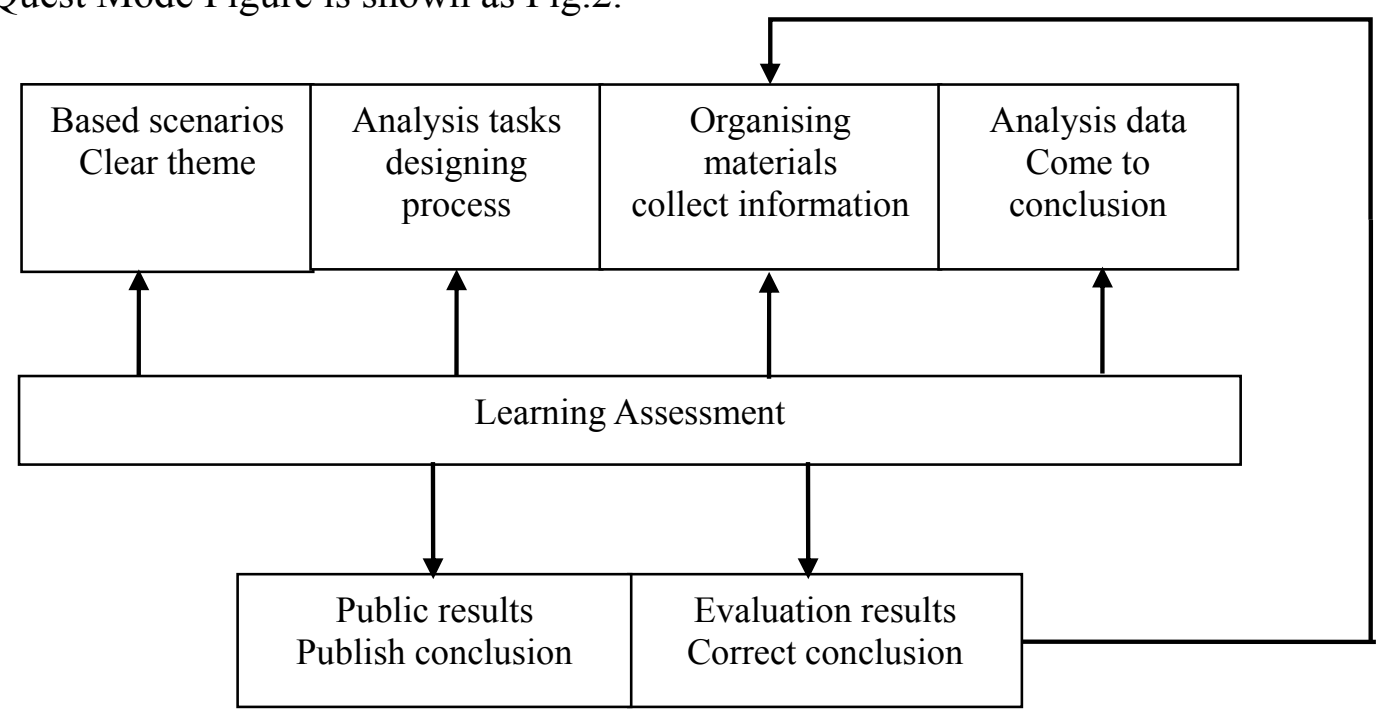

Fig.2 WebQuest Mode Figure

\section{Computer teaching strategies based on network teaching mode WebQuest}

Teaching principles and steps. WebQuest teaching model build a holistic learning activities Wizard for teachers and students. In this paper, base on the five principles proposed by Bernie 
Dodget ${ }^{[1]}$ : providing a targeted site; the effective integration of information and knowledge learners; encouraging students to explore and think; moderating use of various types of media; taking into account the part of the higher expectations of learners.Combined vision Bernie Dodget, based on WebQuest, teaching mode steps are:guiding the students familiar with the contents of the computer network to explore online resources; in-depth integration of these resources, making it easy to query data references; suitable for extracting topic from a computer network curriculum resources; construction of the actual situation of the task for students from selected topics;designing effect learner assessment criteria.

Step in the implementation of teaching, we should pay attention to reflect the students in the classroom learning the subject and encourage students to explore the initiative to collect analytical information, test hypotheses, construct the meaning of knowledge. Also note that the role of teachers converted. Teachers are guides and organizers, its leading role is to stimulate students' motivation to learn, which is the traditional teaching model has a quite different, but also the meaning and value of WebQuest teaching mode where ${ }^{[2]}$.

Course characteristics and student characteristics analysis. "Computer network" is a concept of course involves a lot, considering the current computer field is constantly evolving and updating course content must be able to keep it up. "Computer Network" teaching goals and the effect should be positioned in the network to enable students to understand the basic theory of juice and network protocol, based on this understanding and proficiency in network-related operations, familiar with TCP / IP protocol suite, and to develop practical ability to work with ability.

"Computer Network" program as it relates to a lot of theoretical knowledge, and therefore relatively boring to master some of the teachers if scripted, students of memory caused by boredom, resulting in a class and do not concentrate or even skipping. Due to the large course difficulty, students after absences is difficult to keep up, it is difficult to understand the basics of networking, it is difficult to apply knowledge to solve network problems in real situations. Furthermore, since the source of students to pay school complex, resulting in uneven quality of basic skills and students, there are some students less capable hands, enthusiasm and initiative is not high.

Teaching mode theme selection. Although WebQuest is a new concept of teaching, but only select the appropriate teaching theme, only more conducive to student understanding and mastery. In the choice of the theme of teaching mode, first WebQuest applied within the syllabus more integrated activities, while providing a wealth of information resources for these topics, and as far as possible in this teaching model to guide students to explore the need for a deep understanding and topics examined. Based on this consideration, the teaching focus located in the "Computer Networks" course "network planning and design," which is part of the knowledge of the basics of network construction, the need to give students from the physical layout of the network on the basis of the needs analysis the package architecture of the network, it is possible to greatly exercise its ability to use hands-on ability and comprehensive knowledge of ${ }^{[3]}$.

Specific case study. In this paper, we chose the campus network planning and design study as an example. Due to a larger workload, the author will refine into a number of self-contained sub-tasks, namely: knowledge and status of the investigation during the program planning should have the task team each character must be carried out, and the group of mutual cooperation between tasks. To accomplish these tasks, encourage students to explore the Internet, library access to information, and network status fieldwork.

Through the grasp of the campus network knowledge, this paper think from the following aspects: what is difference and connection between the campus network planning and design? Why the need for network planning and design needs analysis? how to develop the need of analysis outline? When the network cabling can be further subdivided into several subsystems? How the network performance testing and evaluation? What are the main indicators of the test? The success of the campus network to collect some programs from the Internet, features and advantages of the program are summarized compare their use of technology. 
After the students into groups, each group arranged for each role you need to complete the task. Grouping principle "between the two groups homogeneous, heterogeneous within the group."make 4 persons as group. At last, his report makes the PPT for presentation and communication.

\section{Conclusion}

Combining teaching practice, elaborate computer network curriculum teaching model based on WebQuest. This model is conducive to optimizing teaching methods and student learning efficiencies. Construction of learning tasks and contexts through WebQuest model, through massive learning resources and an objective evaluation method, greatly enhance student learning initiative, and in large part enhance the students' enthusiasm for learning, so that to strengthen collaborative learning the ability to cooperate and self-learning ability, to promote the improvement of students' skills.

\section{References}

[1] K. M. Li. WebQuests Teaching Mode Network .J. Hulan Teachers College, 2 (2010) 83-84.

[2] K. C. Yang. Thoughts on constructivist learning theory teaching revelation.J. Educational study, 2(2009) 93-95.

[3] W. Xiang. Practice study on University Computer Foundation Teaching model.J. ARTS AND SCIENCE, 2 (2010) 119-121. 